Check for updates

Cite this: Chem. Sci., 2017, 8, 7457

\title{
Self-quenched ferrocenyl diketopyrrolopyrrole organic nanoparticles with amplifying photothermal effect for cancer therapy $\dagger$
}

\author{
Pingping Liang, ${ }^{a}$ Qianyun Tang, ${ }^{a}$ Yu Cai, ${ }^{a}$ Gongyuan Liu, ${ }^{a}$ Weili Si, ${ }^{a}$ Jinjun Shao, (DD a \\ Wei Huang, ${ }^{* a}$ Qi Zhang*b and Xiaochen Dong (D) *a
}

\begin{abstract}
Organic nanoparticles (NPs) with near-infrared absorbance possess high photothermal conversion (PTC) efficiency and an excellent photoacoustic signal, presenting a great prospect for photoacoustic imaging (PAI)-guided photothermal therapy (PTT). Herein, a novel diketopyrrolopyrrole derivative (DPPCN-Fc) is synthesized for use as a PTT agent with PAI performance. Due to photo-induced electron transfer (PET), the two flanked ferrocene moieties significantly quench the radiative decay and intersystem crossing process, resulting in an enhanced nonradiative transition, and an amplifying photothermal effect is observed. Exposing the DPPCN-Fc NP aqueous dispersion $\left(100 \mu \mathrm{g} \mathrm{m}^{-1}\right)$ to $730 \mathrm{~nm}\left(1.0 \mathrm{~W} \mathrm{~cm}^{-2}\right)$ laser radiation results in a temperature elevation of $33.4^{\circ} \mathrm{C}$ within $10 \mathrm{~min}$ and the PTC efficiency reaches up to $59.1 \%$, which is higher than most reported photothermal therapeutic agents. Furthermore, under irradiation from $730 \mathrm{~nm}$ lasers, cancer cells could be completely killed in vivo due to the amplifying photothermal effects. Therefore, the as-prepared DPPCN-FC NPs are a promising cancer theranostic agent for photoacoustic imaging-guided cancer photothermal therapy.
\end{abstract}

Received 1st August 2017

Accepted 4th September 2017

DOI: $10.1039 / \mathrm{c} 7 \mathrm{sc} 03351 \mathrm{f}$

rsc.li/chemical-science

demand to monitor the distribution of photothermal agents and

\section{Introduction}

Cancer has become one of the major threats to human health in modern society, although great effort has been made toward its treatment. ${ }^{1}$ Traditional treatments, including surgery, hormone therapy, immunotherapy, radiotherapy and chemotherapy, all have their intrinsic limits, such as drug resistance, side effects, low targeting and lack of specificity for individual cancer patients. $^{2-7}$ Photothermal therapy (PTT), a highly selective and noninvasive therapeutic method with near-infrared (NIR) laserinduced ablation of tumor cells, has recently emerged among the many treatments to show great promise for cancer treatment and accordingly has attracted great attention in recent years. ${ }^{8-12}$ However, most photothermal agents are limited by their poor biocompatibility, larger size, superficial penetration depth and low photothermal conversion (PTC) efficiency. ${ }^{13}$ PTT reagents, which possess high PTC efficiency, can reduce the pain and treatment duration experienced by the patients. ${ }^{14}$ In addition, an appropriate imaging technique is in urgent

${ }^{a}$ Key Laboratory of Flexible Electronics (KLOFE), Institute of Advanced Materials (IAM), Jiangsu National Synergetic Innovation Center for Advanced Materials (SICAM), Nanjing Tech University (NanjingTech), 30 South Puzhu Road, Nanjing 211816, China.E-mail: iamxcdong@njtech.edu.cn; iamwhuang@njtech.edu.cn

${ }^{b}$ School of Pharmaceutical Sciences, Nanjing Tech University (NanjingTech), Nanjing, China.E-mail: zhangqi@njtech.edu.cn

$\uparrow$ Electronic supplementary information (ESI) available: Experimental methods and additional figures. See DOI: 10.1039/c7sc03351f to evaluate the therapeutic effect during therapy in vivo. ${ }^{13}$ Photoacoustic imaging (PAI), which can image tissue with a depth of 5-7 cm in a localized region, ${ }^{15-18}$ has higher resolution compared to other traditional optical imaging techniques. PAI is based on the detection of ultrasound waves generated from the thermally induced vibration and expansion of light absorbing tissues under specific pulsed laser irradiation. ${ }^{19,20}$ Furthermore, PAI has the ability to analyze nanomaterial accumulation and blood vessel distribution in tumors. ${ }^{21}$ Hence, the development of a PTT agent with intense NIR (700-1000 nm) absorption as well as PAI performance is necessary for cancer therapy.

Although a large number of NIR absorbing inorganic nanomaterials (such as Au, Ag, Pt, Pd, and C) have been explored for PTT, ${ }^{22-28}$ the potential toxicity and non-biodegradable nature of these inorganic nanostructures have seriously impeded their clinical application. ${ }^{28,29}$ To overcome these problems, a variety of organic photothermal agents have emerged, such as NIR dyes (ICG, IR-820, and IR-825) and RC-BSA. ${ }^{30-32}$ However, the low PTC efficiencies of these organic materials are not satisfactory for clinical application. Hence, the rational design and synthesis of effective PTT agents with higher PTC efficiency is in urgent need.

The diketopyrrolopyrrole (DPP) unit is an excellent electron acceptor due to its lactam structure, which has already been widely used in organic optical materials due to its excellent light absorption property. ${ }^{33-36}$ Recently, triphenylamine based thienyl-diketopyrrolopyrrole (DPP-TPA) with a donor-acceptordonor (D-A-D) structure has shown great promise as 
a therapeutic agent for PTT with a PTC efficiency of $34.5 \%$ as reported by our group. ${ }^{35}$ To further improve the PTC efficiency, furyl-diketopyrrolopyrrole derivatives have come into our view. The steric hindrance of furyl-diketopyrrolopyrrole is smaller than that of thienyl-diketopyrrolopyrrole, and will result in better coplanarity. ${ }^{37,38}$ Furthermore, ferrocene (Fc), a cancer therapeutic agent itself, is introduced into the structure..$^{39-41}$ As a good electron-donating unit, Fc can quench the fluorescence and singlet-oxygen $\left({ }^{1} \mathrm{O}_{2}\right)$ production through a photo-induced electron transfer (PET) process, enhancing the nonradiative transition of thermal energy release. ${ }^{\mathbf{4 1 , 4 2}}$ Besides, it has been proven that iron-containing nanoparticles will be an extra boon for applications in PTT. $^{43}$ Lastly, tetracyanobutadiene (TCBD), a strong electron acceptor unit, is introduced into the material system to build the D-A-D structure, which will give more redshifted absorbance and promote the PET process. ${ }^{23,44-46}$

Herein, an excellent performance furyl-diketopyrrolopyrrole based PTT agent with a high molar extinction coefficient $\varepsilon=$ $3.03( \pm 0.2) \times 10^{4} \mathrm{M}^{-1} \mathrm{~cm}^{-1}$ (at $733 \mathrm{~nm}$ ) and PAI effect in the form of nanoparticles has been designed and synthesized. The obtained Fc flanked DPP derivative with a TCBD unit (DPPCNFc, Scheme 2) shows intense NIR absorption for the PAIguided PTT of cancer. To make the NIR absorbing DPPCN-Fc disperse in water, a re-precipitation method is applied to realize the hydrophilic DPPCN-Fc NPs. ${ }^{47}$ The as-prepared DPPCN-Fc NPs show amplifying PTC efficiency $(\eta=59.1 \%)$, superb stability, good biocompatibility, low dark cytotoxicity as well as excellent PAI property. The process of PET in the DPPCNFc molecule and a simplified representation of the PAI-guided PTT in vivo of DPPCN-Fc NPs are shown in Scheme 1. By carefully designing the structure, DPPCN-Fc NPs are expected to be a high performance therapeutic agent for photoacoustic imaging-guided cancer photothermal therapy.

\section{Results and discussions}

As shown in Scheme 2, DPPBr was synthesized in the presence of $\mathrm{N}$-bromosuccinimide (NBS) in chloroform, then a Pd-

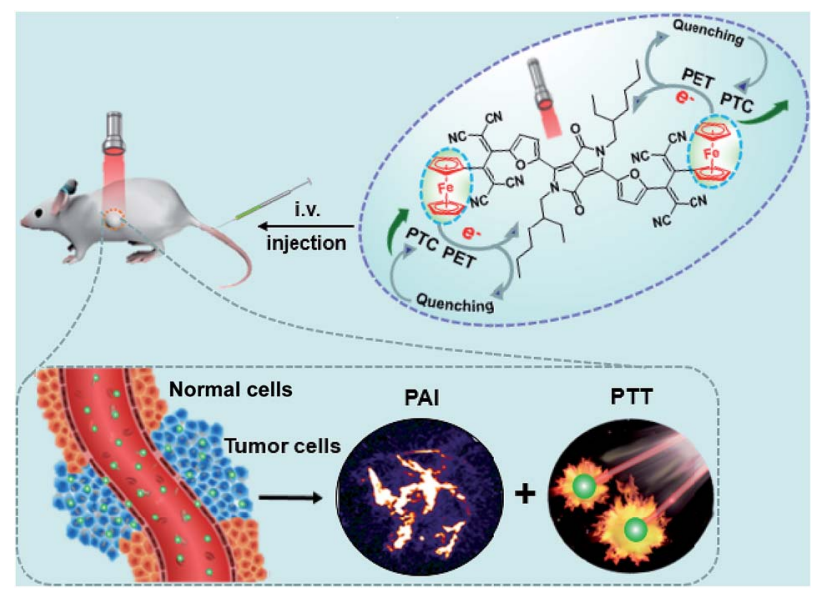

Scheme 1 A simplified representation of the photoacoustic imagingguided photothermal therapy in vivo of DPPCN-Fc NPs. catalyzed Sonogashira cross-coupling reaction was performed to prepare DPP-Fc in good yield. Subsequently, DPPCN-Fc was obtained by a $[2+2]$ cycloaddition reaction with tetracyanoethylene (TCNE). All the intermediates and products were characterized by ${ }^{1} \mathrm{H}$ NMR and ${ }^{13} \mathrm{C}$ NMR. DPPCN-Fc was also characterized by MALDI-TOF-mass spectrometry (Fig. S1-S5 $\dagger$ ). DPPBr, DPP-Fc and DPPCN-Fc are readily soluble in common organic solvents and the respective photographs in dichloromethane (DCM) under daylight are shown in Fig. 1a. The color changes from bluish violet to green. The green dye DPPCN-Fc was then conveniently self-assembled into nanoparticles through $\pi-\pi$ stacking interactions and hydrophobic interactions by the re-precipitation method. ${ }^{47}$ The aqueous dispersion of the DPPCN-Fc nanoparticles is transparent as well (Fig. 1a), which indicates that the DPPCN-Fc NPs present excellent water solubility.

The size and morphology of the DPPCN-Fc NPs were characterized by scanning electron microscopy (SEM) and transmission electron microscopy (TEM) (Fig. 1b), which show welldefined spherical nanostructures. The dynamic light scattering (DLS) measurement further exhibits an average diameter of the DPPCN-Fc NPs as $80 \pm 2.3 \mathrm{~nm}$ with a narrow size distribution (Fig. 1c). Fig. 1d shows that DPP-Fc presents a broad absorption band with $\lambda_{\max }$ at $606 \mathrm{~nm}$ and DPPCN-Fc exhibits a broader absorption band in the visible-NIR region with $\lambda_{\max }$ at $728 \mathrm{~nm}$ with a red-shift of $122 \mathrm{~nm}$ compared to DPP-Fc. This phenomenon comes from the fact that TCNE is a good electronwithdrawing unit, which can construct a D-A structure and push its absorbance to the biological window (700-1000 nm). Moreover, the DPPCN-Fc NPs in water also show a broad absorption band with $\lambda_{\max }$ at $733 \mathrm{~nm}$, caused by the strong $\pi-\pi$ stacking interactions of DPPCN-Fc in the nanoparticle structure. ${ }^{47}$ In addition, the DPPCN-Fc NPs show a high molar extinction coefficient $\left(\varepsilon=3.03( \pm 0.2) \times 10^{4} \mathrm{M}^{-1} \mathrm{~cm}^{-1}\right.$, calculated by $A=\varepsilon \times C \times L$ ) at $733 \mathrm{~nm}$ (Fig. S6 $\dagger$ ), which demonstrates the strong light absorption ability of the DPPCN-Fc NPs.

The intensive NIR absorption in the NIR region suggests that the DPPCN-Fc NPs would be an excellent photo-absorbing agent for PTT. To examine the photothermal effect of the DPPCN-Fc NPs, aqueous dispersions with different concentrations at 0 , $20,40,60,80$, and $100 \mu \mathrm{g} \mathrm{mL}{ }^{-1}$ were exposed to a $730 \mathrm{~nm}$ NIR laser at a power density of $1.0 \mathrm{~W} \mathrm{~cm}^{-2}$. As shown in Fig. 2a, the temperature increase of the DPPCN-Fc NPs is obviously concentration-dependent under laser irradiation. At the concentration of $100 \mu \mathrm{g} \mathrm{mL} \mathrm{m}^{-1}$, the temperature elevation is $33.4{ }^{\circ} \mathrm{C}$. In comparison, no obvious temperature increase was

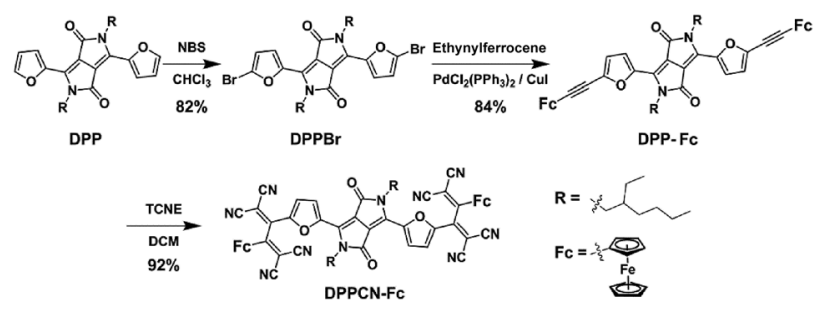

Scheme 2 Synthetic route to DPPCN-Fc. 
(a)
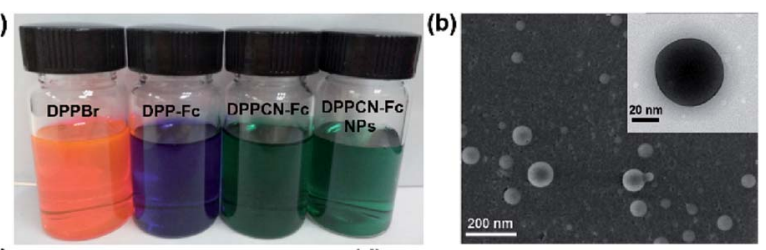

(c)
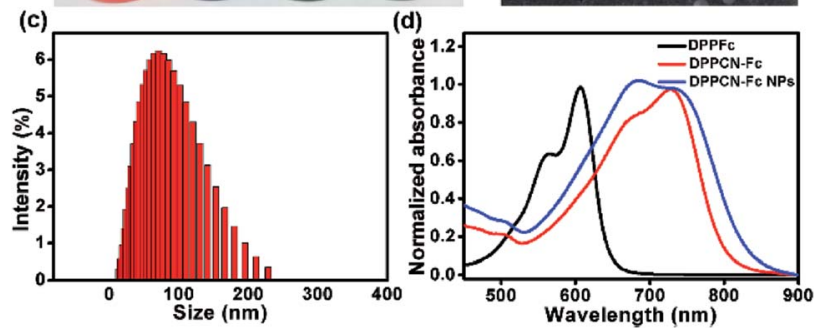

Fig. 1 (a) Photographs of DPPBr, DPP-Fc, and DPPCN-Fc in DCM, and the DPPCN-FC NPs in water. (b) A SEM image of the DPPCN-FC NPs. Inset: a TEM image of a single DPPCN-FC NP. (c) The DLS size distribution of the DPPCN-Fc NPs. (d) The normalized absorbance spectra of DPP-FC, DPPCN-FC in DCM, and the DPPCN-FC NPs in water.
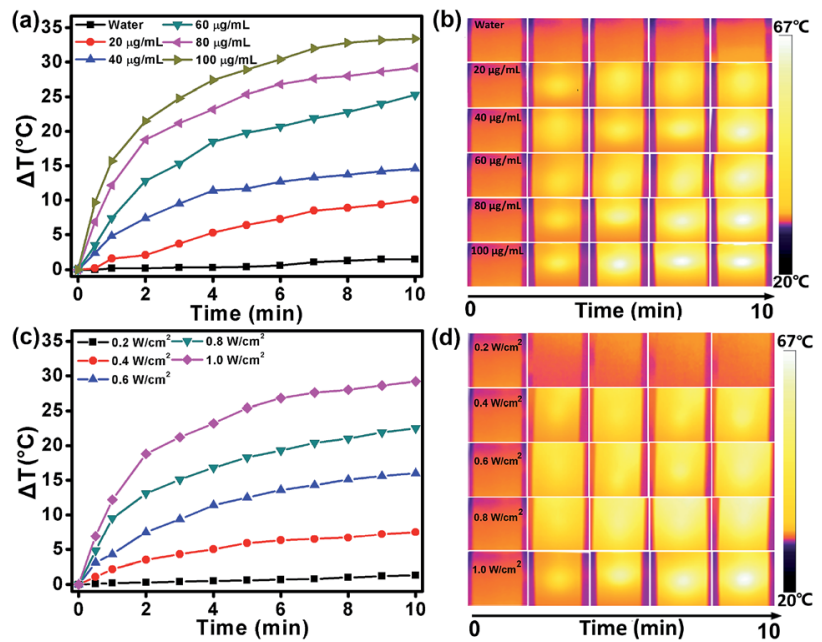

Fig. 2 ( $a$ and $c$ ) The heating curves of the DPPCN-Fc NPs aqueous dispersions with different concentrations $(0,20,40,60,80$, and $100 \mu \mathrm{g}$ $\mathrm{mL}^{-1}$ ) at a power density of $1.0 \mathrm{~W} \mathrm{~cm}^{-2}$ and various power densities $\left(0.2,0.4,0.6,0.8\right.$, and $\left.1.0 \mathrm{~W} \mathrm{~cm}^{-2}\right)$ at a concentration of $80 \mu \mathrm{g} \mathrm{mL}^{-1}$ (730 $\mathrm{nm}$ laser, $10 \mathrm{~min}$ ). (b and d) The IR thermal images of the DPPCNFc NPs aqueous dispersions with various concentrations and power densities (730 nm laser, $10 \mathrm{~min}$ ).

observed for pure deionized water under the same conditions, demonstrating the excellent photothermal effect of the DPPCNFc NPs. When the DPPCN-Fc NP dispersion at a concentration of $80 \mu \mathrm{g} \mathrm{mL} \mathrm{m}^{-1}$ was exposed to a $730 \mathrm{~nm}$ NIR laser with power densities at $0.2,0.4,0.6,0.8$ and $1.0 \mathrm{~W} \mathrm{~cm}^{-2}$, the temperature increase showed an obvious laser power intensity dependence. At a power density of $1.0 \mathrm{~W} \mathrm{~cm}^{-2}$, the temperature elevation is $29.2^{\circ} \mathrm{C}$, as shown in Fig. 2c. Moreover, the infrared (IR) thermal images (Fig. 2b and d, recorded by an IR camera) show an obvious colour change from dark to white, which further confirms the excellent photothermal performance of the DPPCN-Fc NPs. All these results suggest that the DPPCN-Fc NPs are promising PTT agents with good PTC efficiency.
To further estimate the light-to-heat conversion of the nonradiative decay, the PTC efficiency $(\eta)$ of the DPPCN-Fc NPs was calculated according to the equation described in the ESI. $\uparrow$ The temperature changes of the DPPCN-Fc NPs in the aqueous dispersion were recorded under $730 \mathrm{~nm}$ laser irradiation for $600 \mathrm{~s}$ and cooling for another $600 \mathrm{~s}$. As a comparison, pure deionized water without the DPPCN-Fc NPs was also irradiated under the same conditions as a control, as shown in Fig. 3a and b. As a result, the PTC efficiency of the DPPCN-Fc NPs is calculated to be $59.1 \%$, which is significantly higher than that of HMPBs (41.4\%), ${ }^{48}$ RC-BSA nanoparticles $(28.7 \%),{ }^{31}$ TPT nanoparticles (32\%), ${ }^{49}$ gold nanovesicles $(37 \%),{ }^{11}$ Au nanorods $(21 \%),{ }^{50} \mathrm{Cu}_{9} \mathrm{~S}_{5}(25.7 \%),{ }^{14}$ and so on. Fig. $3 \mathrm{c}$ shows the light-toheat cycle stability of the DPPCN-Fc nanoparticles. There is no decrease in the temperature elevation after four cycles of alternate heating (laser on: $5 \mathrm{~min}$ ) and cooling (laser off: $5 \mathrm{~min}$ ), which reveals that the DPPCN-Fc NPs have good photothermal stability. After the cycle measurements, the UV-vis-NIR spectrum of the DPPCN-Fc NP dispersion presents almost no obvious change in contrast to that before irradiation, as shown in Fig. 3d. In addition, we further characterized the size and morphology of the DPPCN-Fc NPs by SEM and TEM after laser irradiation to confirm the photostability. To verify whether the dispersant has an effect on the photostability, DPPCN-Fc NPs in phosphate buffer saline (PBS) were also studied. All the results showed that the nanoparticles retained their spherical nanostructures and size, which further revealed that the DPPCN-Fc NPs have excellent photostability in water and PBS (Fig. S7†). The high PTC efficiency and superbly stable photothermal performance of the DPPCN-Fc NPs further makes them a promising photothermal agent for cancer PTT.

The fluorescence decay profiles of DPPBr, DPP-Fc and DPPCN-Fc were measured via the time-correlated single photon
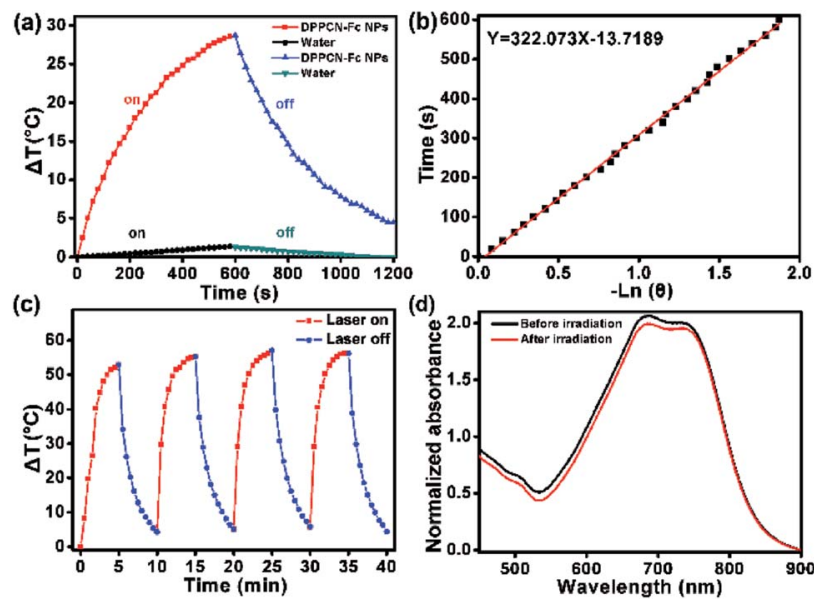

Fig. 3 (a) The heating curve of the DPPCN-Fc NP aqueous dispersion in a procedure of laser-on and off for 20 min (pure water was irradiated as a control). (b) The linear cooling time data versus $-\ln (\theta)$ obtained from the cooling period of (a). (c) The heating curve of the DPPCN-FC $\mathrm{NP}$ aqueous dispersion at a concentration of $200 \mu \mathrm{g} \mathrm{mL}^{-1}$ during four cycles of on-and-off laser irradiation. (d) The UV-vis-NIR absorbance spectra of the DPPCN-Fc NPs before/after laser irradiation $(730 \mathrm{~nm}$, $\left.1.0 \mathrm{~W} \mathrm{~cm}^{-2}, 20 \mathrm{~min}\right)$. 
counting (TCSPC) technique (Fig. S8a †). Mono-exponential decays were observed for these three compounds, and the fluorescence lifetimes are 6.03, 2.08, and $0.17 \mathrm{~ns}$, respectively. A similar result was observed regarding the fluorescence spectra of DPPBr, DPP-Fc and DPPCN-Fc, which show that the fluorescence of DPP-Fc and DPPCN-Fc is mostly quenched (Fig. S8b $\dagger$ ). The lifetime and fluorescence intensity descents of DPPCN-Fc reveal that the two Fc units can effectively quench the fluorescence of the center chromophore through a PET process. In addition, the ${ }^{1} \mathrm{O}_{2}$ production of DPPBr, DPP-Fc and DPPCN-Fc in DCM was measured using 1,3-diphenylisobenzofuran (DPBF) as an indicator. ${ }^{34,51}$ At $411 \mathrm{~nm}$, the degradation times of DPBF were $30 \mathrm{~s}$ and $15 \mathrm{~min}$ for DPPBr and DPP-Fc, respectively. However, the absorbance of DPBF in the DPPCN-Fc solution showed almost no degradation at $411 \mathrm{~nm}$, even though the illumination time was extended up to $15 \mathrm{~min}$ (Fig. S8c $\dagger$ ). The longer degradation time of DPBF demonstrates that less ${ }^{1} \mathrm{O}_{2}$ is generated by DPPCN-Fc via the ISC process. To further confirm the result in cancer cells (nuclei dyed with DAPI), fluorescence images were recorded using $2^{\prime}, 7^{\prime}$-dichlorofluorescein diacetate (DCFH-DA) as a probe to detect ${ }^{1} \mathrm{O}_{2}$ generation in HeLa cells. As shown in Fig. $\mathrm{S} 9, \dagger$ there is no ${ }^{1} \mathrm{O}_{2}$ production after illumination for DPPCN-Fc, which further proves that the two Fc units can effectively quench ${ }^{1} \mathrm{O}_{2}$ generation through a PET process.

According to these measurements and the result of the high PTC efficiency, a PET mechanism was proposed to better understand the amplifying photothermal property of the DPPCN-Fc NPs. As shown in Fig. 4, when DPPCN-Fc is excited from the ground state $\left(S_{0}\right)$ to the excited state $\left(S_{1}\right)$, this electron transfer is supposed to quench the $S_{1}$ state to generate the charge transfer (CT) state. Once the CT state is produced, the electrons will transfer to the CT state, which will quench the radiative decay of fluorescence and release nonradiative energy as heat. In addition, the electrons will not jump to $\mathrm{T}_{1}$ through intersystem crossing (ISC), thereafter resulting in the low photosensitization property of ${ }^{3} \mathrm{O}_{2}$ to ${ }^{1} \mathrm{O}_{2} \cdot{ }^{52}$

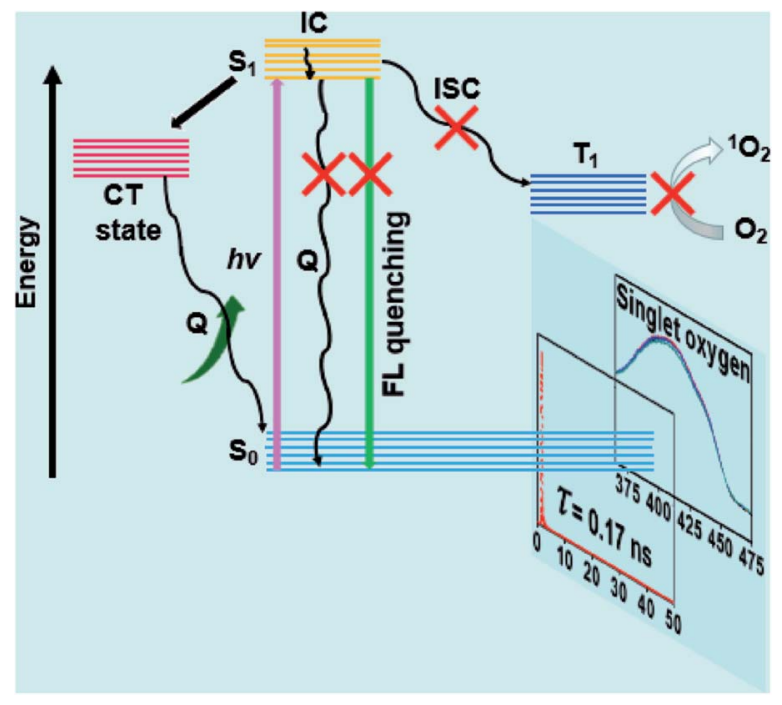

Fig. 4 The proposed PET mechanism for the high PTC efficiency.
Encouraged by the outstanding PTC efficiency, the potential toxicity of the DPPCN-Fc NPs in vitro was further investigated by a cell viability MTT assay on HeLa cells first. It is well known that an ideal photothermal agent should be nontoxic or lowtoxic in vitro or in vivo. ${ }^{\mathbf{1 4}}$ As shown in Fig. 5a, the DPPCN-Fc NPs showed no dark cytotoxicity even at a high concentration $\left(120 \mu \mathrm{g} \mathrm{mL}^{-1}\right)$ after incubation for $24 \mathrm{~h}$ or even $48 \mathrm{~h}$. Furthermore, the photothermal effect of the DPPCN-Fc NPs in vitro was tested by incubating HeLa cells with the DPPCN-Fc NPs and irradiating them under a $730 \mathrm{~nm}$ NIR laser. The viability of the HeLa cells declines with an increasing concentration of the DPPCN-Fc NPs. The $50 \%$ inhibitory concentration $\left(\mathrm{IC}_{50}\right)$ is about $13 \mu \mathrm{g} \mathrm{mL}{ }^{-1}$ (Fig. 5b). The cancer cell line A2780 was also operated as a parallel test, and the result is consistent with that in the HeLa cells. No dark toxicity and the high phototoxicity and good biocompatibility of the DPPCN-Fc NPs indicate that this material has great promise as an effective PTT agent for tumor therapy in vivo.

After confirming the advantages of the DPPCN-Fc NPs in the cellular microenvironment, the practical application for livingcell imaging was investigated, monitored by confocal microscopy after incubating the HeLa cells with the DPPCN-Fc NPs for $12 \mathrm{~h}$ and then staining with 4 $^{\prime}$,6-diamidino-2-phenylindole (DAPI) for nuclei imaging. Fig. 5c shows an obvious intracellular red fluorescence (excited at $488 \mathrm{~nm}$ ). The overlay of the DPPCN-Fc NP fluorescence and nuclei staining indicates that the NPs are predominantly delivered to the cytoplasmic matrix of the cells. This result demonstrates that the DPPCN-Fc NPs present excellent cell uptake performance.

As the DPPCN-Fc NPs possess strong NIR absorption, they would be an excellent agent for PAI.$^{53}$ As shown in Fig. 6a and b, the PA signal linearly increases with the increasing concentration of the DPPCN-Fc NPs. HeLa tumor-bearing mice were selected as the animal model for PAI in vivo, and were treated by
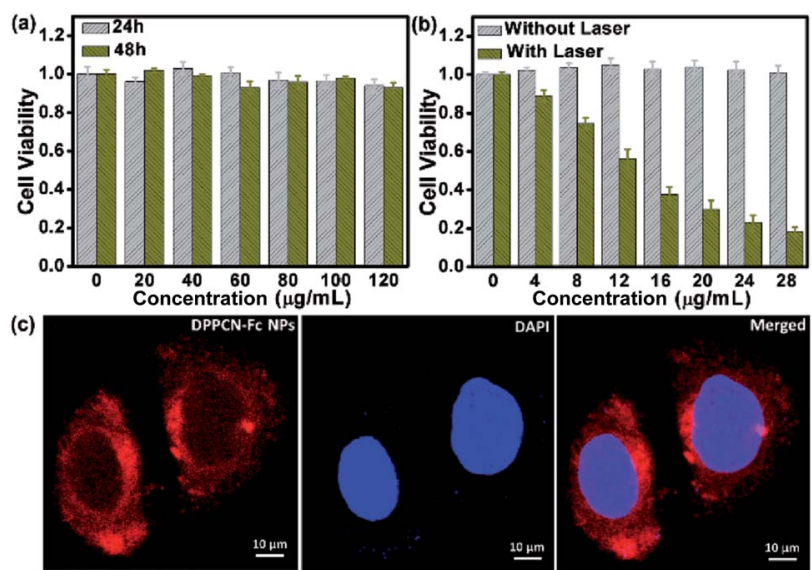

Fig. 5 (a) The cell viability of HeLa cells incubated with the DPPCN-FC NPs at different concentrations and times in dark conditions. (b) The cell viability of HeLa cells incubated with the DPPCN-Fc NPs without and with irradiation by a $730 \mathrm{~nm}$ laser $\left(1.0 \mathrm{~W} \mathrm{~cm}^{-2}, 3 \mathrm{~min}\right)$. (c) Confocal microscopy images of the HeLa cells incubated with the DPPCN-Fc NPs $\left(80 \mu \mathrm{g} \mathrm{mL}^{-1}\right)$ for 6 hours. The red and blue colours represent the fluorescence (excited at $488 \mathrm{~nm}$ ) of the DPPCN-FC NPs and DAPI images, respectively. 
intravenous injections with the DPPCN-Fc NPs $\left(80 \mu \mathrm{g} \mathrm{mL}^{-1}, 100\right.$ $\mu \mathrm{L})$. Compared to the pre-injection image, the PA signals induced by the DPPCN-Fc NPs increased and reached a maximum at $6 \mathrm{~h}$ (Fig. $6 \mathrm{c}$ and $\mathrm{d}$ ). Then the PA signals decreased gradually. This result indicates the efficient accumulation of the DPPCN-Fc NPs in the tumor site owing to the enhanced permeability and retention (EPR) effect, ${ }^{54}$ which reveals that the DPPCN-Fc NPs present a great prospect for PAI-guided PTT.

Motivated by the strong NIR optical absorption, amplifying photothermal performance and excellent PAI properties of the DPPCN-Fc NPs, the cancer photothermal therapy in vivo was measured with mice bearing HeLa tumours intravenously injected with the DPPCN-Fc NPs $\left(100 \mu \mathrm{L}, 80 \mu \mathrm{g} \mathrm{mL}^{-1}\right)$ and saline $(100 \mu \mathrm{L}$, as the control). After $6 \mathrm{~h}$ of injection, the tumors on these mice were exposed to a $730 \mathrm{~nm}$ laser at a power density of $1.0 \mathrm{~W} \mathrm{~cm}{ }^{-2}$ for $8 \mathrm{~min}$. Under irradiation, the tumor surface temperature increased rapidly (monitored by an IR thermal camera) within $8 \mathrm{~min}$, as shown in Fig. 7a. This result can be attributed to the high tumor accumulation and the superb photothermal effect of the DPPCN-Fc NPs. In contrast, the tumor temperature without the DPPCN-Fc NP injection shows little change under the same irradiation conditions. To further estimate the PTT of the DPPCN-Fc NPs in vivo, HeLa tumorbearing mice were randomly divided into 4 groups $(n=5)$ : (i) saline without irradiation as the control group, (ii) saline with irradiation, (iii) the DPPCN-Fc NPs without irradiation, and (iv) the DPPCN-Fc NPs with irradiation as the treatment group. All the mice were treated by intravenous injection every two days. ${ }^{55}$ In the treatment group, all the tumours were completely ablated at the secondary treatment, then the black scars fell off at the tumor sites and no recurrence was observed. In marked contrast, the tumor volumes of all the mice in the other three groups reached up to $2000 \mathrm{~mm}^{3}$ within the life span of 18 days, as shown in Fig. 7b. The visual photograph of the therapeutic result is shown in Fig. 7d. The tumor of the treatment group was eliminated completely, suggesting the excellent photothermal effect of the DPPCN-Fc NPs.
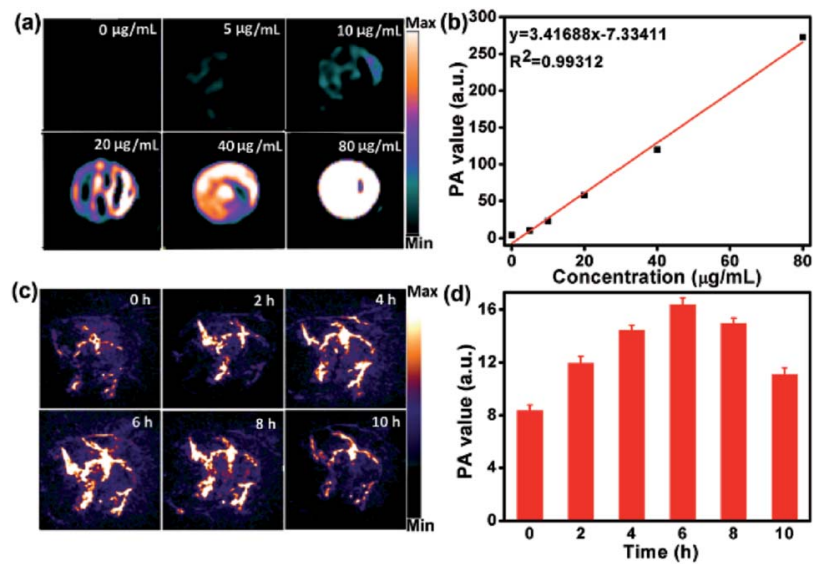

Fig. 6 ( $a$ and $b$ ) The PA images and signal values of the DPPCN-Fc NP aqueous dispersions with different concentrations. (c and d) The PA images and signal values of the HeLa tumor on mice obtained at different times after i.v. injection of the DPPCN-Fc NPs $\left(80 \mu \mathrm{g} \mathrm{mL}^{-1}\right)$.

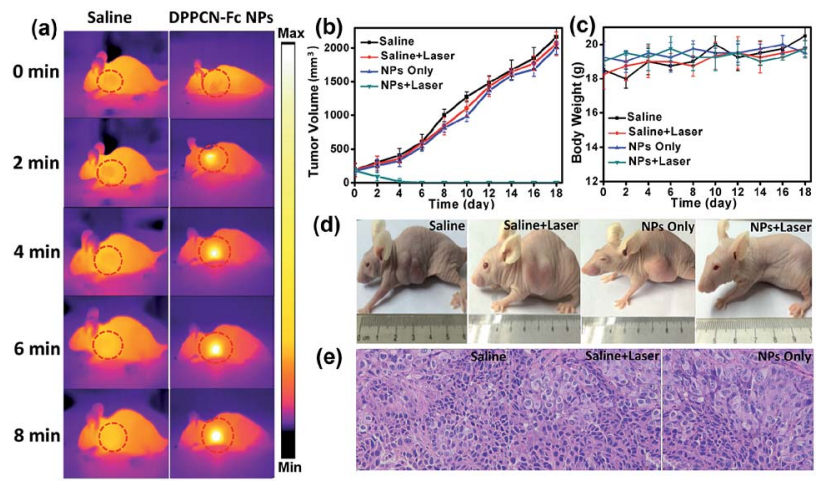

Fig. 7 (a) The IR thermal images of HeLa tumor-bearing mice with and without the DPPCN-FC NPs $\left(80 \mu \mathrm{g} \mathrm{mL}^{-1}, 100 \mu \mathrm{L}\right)$ under $730 \mathrm{~nm}$ laser irradiation at a power density of $1.0 \mathrm{~W} \mathrm{~cm}^{-2}$ for $8 \mathrm{~min}(6 \mathrm{~h}$ after injection). (b and c) The tumor volume and body weight changes of the four groups during treatment. Each data represents the mean of quintuplicate measurements. (d) Photographs of the HeLa tumorbearing mice after treatment with the DPPCN-Fc NPs. (e) Photographs of the H\&E stained tumor tissues obtained from the different groups after 18 days treatment.

Although the DPPCN-Fc NPs have no dark toxicity in vitro, the potential toxicity in vivo is still an important issue to be evaluated. As expected, no abnormalities in body weight (Fig. 7c), eating, drinking, urination and activity were observed during the whole treatment process. Furthermore, images of the major organs from the four groups by Hematoxylin and Eosin (H\&E) staining were also recorded, and there are no abnormalities for these organs (heart, liver, spleen, lung, and kidney), as shown in Fig. S11. $\dagger$ Moreover, the tumors of groups (i), (ii), and (iii) were also stained by H\&E after the mice were sacrificed to investigate the therapeutic effects. The tumor cells of these three groups are arranged closely and the nuclei of the tumor cells are in good condition, as shown in Fig. 7e. All these results indicate little side effects and the minimally invasive character of the DPPCN-Fc NPs, which further confirms that the DPPCN-Fc NPs can be used as a potential therapeutic agent for PTT.

\section{Conclusions}

In conclusion, near-infrared DPPCN-Fc NPs have been designed and synthesized, in which two ferrocene units significantly quench the fluorescence and intersystem crossing because of photo-induced electron transfer (PET), which results in amplifying heat release. As a result, a high photothermal conversion efficiency of $59.1 \%$ was obtained. Furthermore, with the targeted accumulation of the DPPCN-Fc NPs in tumor tissues by the EPR effect in vivo, excellent PAI signals could be clearly observed at 6 hours after intravenous injection. The DPPCN-Fc NP agent showed an excellent cancer cell killing effect at injection doses as low as $0.4 \mathrm{mg} \mathrm{kg}^{-1}$ without any side effects in vivo. Accordingly, the as-prepared DPPCN-Fc NPs are a promising therapeutic agent for photoacoustic imaging-guided cancer photothermal therapy. 


\section{Conflicts of interest}

The authors of this manuscript have no conflicts of interest.

\section{Acknowledgements}

The work was supported by the NNSF of China (61525402, 61775095, 61604071), Key University Science Research Project of Jiangsu Province (15KJA430006), and Natural Science Foundation of Jiangsu Province (BK20161012).

\section{References}

1 M. Ferrari, Nat. Rev. Cancer, 2005, 5, 161-171.

2 N. Rozanova and J. Zhang, Sci. China, Ser. B: Chem., 2009, 52, 1559-1575.

3 H. Chen, F. Liu, Z. Lei, L. Ma and Z. Wang, RSC Adv., 2015, 5, 84980-84987.

4 D. Peer, J. M. Karp, S. Hong, O. C. Farokhzad, R. Margalit and R. Langer, Nat. Nanotechnol., 2007, 2, 751-760.

5 M. Ibrahim, R. Sabouni and G. A. Husseini, Curr. Med. Chem., 2017, 24, 193-214.

6 C. Wang, Y. Ye, G. M. Hochu, H. Sadeghifar and Z. Gu, Nano Lett., 2016, 16, 2334-2340.

7 C. Wang, W. Sun, Y. Ye, Q. Hu, H. N. Bomba and Z. Gu, Nat. Biomed. Eng., 2017, 1, 0011.

8 J. Wang, R. Yan, F. Guo, M. Yu, F. Tan and N. Li, Nanotechnology, 2016, 27, 285102.

9 M. Sun, F. Liu, Y. Zhu, W. Wang, J. Hu, J. Liu, Z. Dai, K. Wang, Y. Wei and J. Bai, Nanoscale, 2016, 8, 4452-4457.

10 P. Huang, Y. Gao, J. Lin, H. Hu, H.-S. Liao, X. Yan, Y. Tang, A. Jin, J. Song and G. Niu, ACS Nano, 2015, 9, 9517-9527.

11 P. Huang, J. Lin, W. Li, P. Rong, Z. Wang, S. Wang, X. Wang, X. Sun, M. Aronova and G. Niu, Angew. Chem., 2013, 125, 14208-14214.

12 X. Huang, P. K. Jain, I. H. El-Sayed and M. A. El-Sayed, Laser Med. Sci., 2008, 23, 217-228.

13 S. Tang, M. Chen and N. Zheng, Nano Res., 2015, 8, 165-174. 14 Q. Tian, F. Jiang, R. Zou, Q. Liu, Z. Chen, M. Zhu, S. Yang, J. Wang, J. Wang and J. Hu, ACS Nano, 2011, 5, 9761-9771.

15 Q. Fan, K. Cheng, Z. Yang, R. Zhang, M. Yang, X. Hu, X. Ma, L. Bu, X. Lu and X. Xiong, Adv. Mater., 2015, 27, 843-847.

16 A. de la Zerda, S. Bodapati, R. Teed, S. Y. May, S. M. Tabakman, Z. Liu, B. T. Khuri-Yakub, X. Chen, H. Dai and S. S. Gambhir, ACS Nano, 2012, 6, 4694-4701.

17 Y.-S. Chen, W. Frey, S. Kim, P. Kruizinga, K. Homan and S. Emelianov, Nano Lett., 2011, 11, 348-354.

18 C. Kim, E. C. Cho, J. Chen, K. H. Song, L. Au, C. Favazza, Q. Zhang, C. M. Cobley, F. Gao and Y. Xia, ACS Nano, 2010, 4, 4559-4564.

19 K. Wilson, K. Homan and S. Emelianov, Nat. Commun., 2012, 3, 618.

20 S. Mallidi, T. Larson, J. Tam, P. P. Joshi, A. Karpiouk, K. Sokolov and S. Emelianov, Nano Lett., 2009, 9, 2825-2831.

21 G. Wang, F. Zhang, R. Tian, L. Zhang, G. Fu, L. Yang and L. Zhu, ACS Appl. Mater. Interfaces, 2016, 8, 5608-5617.
22 A. M. Gobin, E. M. Watkins, E. Quevedo, V. L. Colvin and J. L. West, Small, 2010, 6, 745-752.

23 D. Wang, Z. Guo, J. Zhou, J. Chen, G. Zhao, R. Chen, M. He, Z. Liu, H. Wang and Q. Chen, Small, 2015, 11, 5956-5967.

24 H. Bi, Y. Dai, J. Xu, R. Lv, F. He, S. Gai, D. Yang and P. Yang, J. Mater. Chem. B, 2016, 4, 5938-5946.

25 M. Chen, S. Chen, C. He, S. Mo, X. Wang, G. Liu and N. Zheng, Nano Res., 2017, 1-15.

26 J. T. Robinson, K. Welsher, S. M. Tabakman, S. P. Sherlock, H. Wang, R. Luong and H. Dai, Nano Res., 2010, 3, 779-793.

27 S. Zhang, K. Yang and Z. Liu, Sci. China: Chem., 2010, 53, 2217-2225.

28 N. Lewinski, V. Colvin and R. Drezek, Small, 2008, 4, 26-49. 29 L. Braydich-Stolle, S. Hussain, J. J. Schlager and M.-C. Hofmann, Toxicol. Sci., 2005, 88, 412-419.

30 X. Song, Q. Chen and Z. Liu, Nano Res., 2015, 8, 340-354. 31 B. Zhou, Y. Li, G. Niu, M. Lan, Q. Jia and Q. Liang, ACS Appl. Mater. Interfaces, 2016, 8, 29899-29905.

32 F. Gao, L. Bai, X. Feng, H. P. Tham, R. Zhang, Y. Zhang, S. Liu, L. Zhao, Y. Zheng and Y. Zhao, Small, 2016, 12, 5239-5244.

33 Y. Cai, Q. Tang, X. Wu, W. Si, W. Huang, Q. Zhang and X. Dong, ChemistrySelect, 2016, 1, 3071-3074.

34 Y. Cai, Q. Tang, X. Wu, W. Si, Q. Zhang, W. Huang and X. Dong, ACS Appl. Mater. Interfaces, 2016, 8, 10737-10742.

35 Y. Cai, P. Liang, Q. Tang, X. Yang, W. Si, W. Huang, Q. Zhang and X.-C. Dong, ACS Nano, 2017, 11, 1054-1063.

36 Y. Cai, W. Si, Q. Tang, P. Liang, C. Zhang, P. Chen, Q. Zhang, W. Huang and X. Dong, Nano Res., 2017, 10, 794-801.

37 H. Zhu, W. Huang, Y. Huang, J. Yang and W. Wang, Dyes Pigm., 2016, 127, 37-44.

38 E. Ripaud, D. Demeter, T. Rousseau, E. Boucard-Cétol, M. Allain, R. Po, P. Leriche and J. Roncali, Dyes Pigm., 2012, 95, 126-133.

39 X. J. Jiang, J. T. Lau, Q. Wang, D. K. Ng and P. C. Lo, Chem.Eur. J., 2016, 22, 8273-8281.

40 C. Ornelas, New J. Chem., 2011, 35, 1973-1985.

41 J. T. Lau, X.-J. Jiang, D. K. Ng and P.-C. Lo, Chem. Commun., 2013, 49, 4274-4276.

42 J. F. Lovell, J. Chen, M. T. Jarvi, W.-G. Cao, A. D. Allen, Y. Liu, T. T. Tidwell, B. C. Wilson and G. Zheng, J. Phys. Chem. B, 2009, 113, 3203-3211.

43 S. Ghosh, T. Avellini, A. Petrelli, I. Kriegel, R. Gaspari, G. Almeida, G. Bertoni, A. Cavalli, F. Scotognella and T. Pellegrino, Chem. Mater., 2016, 4848-4858.

44 P. S. Bhale, H. V. Chavan, S. B. Dongare, S. N. Shringare, Y. B. Mule, S. S. Nagane and B. P. Bandgar, Bioorg. Med. Chem. Lett., 2017, 27, 1502-1507.

45 K. Li, L. Li, S. Wang, X. Li, T. Ma, D. Liu, Y. Jing and L. Zhao, Eur. J. Med. Chem., 2017, 126, 910-919.

46 B. Dhokale, T. Jadhav, S. M. Mobin and R. Misra, Dalton Trans., 2016, 45, 1476-1483.

47 M. Lan, J. Zhang, X. Zhu, P. Wang, X. Chen, C.-S. Lee and W. Zhang, Nano Res., 2015, 8, 2380-2389.

48 X. Cai, X. Jia, W. Gao, K. Zhang, M. Ma, S. Wang, Y. Zheng, J. Shi and H. Chen, Adv. Funct. Mater., 2015, 25, 2520-2529. 
49 T. Sun, J. Qi, M. Zheng, Z. Xie, Z. Wang and X. Jing, Colloids Surf., 2015, 136, 201-206.

50 Y. Chen, H. Chen, D. Zeng, Y. Tian, F. Chen, J. Feng and J. Shi, ACS Nano, 2010, 4, 6001-6013.

51 H. Shi, W. Sun, C. Liu, G. Gu, B. Ma, W. Si, N. Fu, Q. Zhang, W. Huang and X. Dong, J. Phys. Chem. B, 2016, 4, 113-120.

52 H. Horiuchi, R. Kuribara, A. Hirabara and T. Okutsu, J. Phys. Chem. A, 2016, 120, 5554-5561.
53 X. Tan, J. Wang, X. Pang, L. Liu, Q. Sun, Q. You, F. Tan and N. Li, ACS Appl. Mater. Interfaces, 2016, 34991-35003.

54 L. Cheng, K. Yang, Q. Chen and Z. Liu, ACS Nano, 2012, 6, 5605-5613.

55 S. Xu, Y. Yuan, X. Cai, C.-J. Zhang, F. Hu, J. Liang, G. Zhang, D. Zhang and B. Liu, Chem. Sci., 2015, 6, 5824-5830. 